\title{
BMJ Open Development and validation of a patient-reported measure of compassion in healthcare: the Sinclair Compassion Questionnaire (SCQ)
}

To cite: Sinclair S,

Hack TF, Maclnnis CC, et al. Development and validation of a patient-reported measure of compassion in healthcare: the Sinclair Compassion Questionnaire (SCQ). BMJ Open 2021;11:e045988. doi:10.1136/ bmjopen-2020-045988

- Prepublication history and additional supplemental material for this paper are available online. To view these files, please visit the journal online (http://dx.doi.org/10.1136/ bmjopen-2020-045988).

Received 26 0ctober 2020 Accepted 06 June 2021

Check for updates

(C) Author(s) (or their employer(s)) 2021. Re-use permitted under CC BY-NC. No commercial re-use. See rights and permissions. Published by BMJ.

For numbered affiliations see end of article.

\section{Correspondence to} Dr Shane Sinclair, Compassion Research Lab, Faculty of Nursing, University of Calgary, Calgary, Alberta, Canada; sinclair@ucalgary.ca

\section{ABSTRACT}

Objectives Compassion is a key indicator of quality care that is reportedly eroding from patients' care experience. While the need to assess compassion is recognised, valid and reliable measures are lacking. This study developed and validated a clinically informed, psychometrically rigorous, patient-reported compassion measure.

Design Data were collected from participants living with life-limiting illnesses over two study phases across four care settings (acute care, hospice, long term care (LTC) and homecare). In phase 1, data were analysed through exploratory factor analysis (EFA), with the final items analysed via confirmatory factor analysis (CFA) in phase 2. The Schwartz Center Compassionate Care Scale (SCCCS), the revised Edmonton Symptom Assessment Scale (ESAS-r) and Picker Patient Experience Questionnaire (PPEQ) were also administered in phase 2 to assess convergent and divergent validity.

Setting and participants 633 participants were recruited over two study phases. In the EFA phase, a 54-item version of the measure was administered to 303 participants, with 330 participants being administered the final 15-item measure in the CFA phase.

Results Both EFA and CFA confirmed compassion as a single factor construct with factor loadings for the 15item measure ranging from 0.76 to 0.86 , with excellent test-retest reliability (intraclass correlation coefficient range: $0.74-0.89$ ) and excellent internal reliability (Cronbach's alpha of 0.96). The measure was positively correlated with the SCCCS $(r=0.75, p<0.001)$ and PPEQ $(r=0.60, p<0.001)$. Participants reporting higher experiences of compassion had significantly greater well-being and lower depression on the ESAS-r. Patients in acute care and hospice reported significantly greater experiences of compassion than LTC residents.

Conclusions There is strong initial psychometric evidence for the Sinclair Compassion Questionnaire (SCQ) as a valid and reliable patient-reported compassion measure. The SCQ provides healthcare providers, settings and administrators the means to routinely measure patients experiences of compassion, while providing researchers a robust measure to conduct high-quality research.

\section{Strengths and limitations of this study}

- This study overcomes the inherent limitations of pre-existing patient-reported compassion measures including but not limited to, insufficient evidence of internal consistency; validity; test accuracy; reliability and sensitivity.

- The Sinclair Compassion Questionnaire (SCQ) was developed in accordance with strict measure development guidelines; by engaging patients across each study stage and from an empirical model of the construct of interest, producing exploratory factor analysis and confirmatory factor analysis results that indicate it has strong reliability and validity evidence as a patient-reported compassion measure.

- While it is anticipated that the SCQ is adaptable to other settings, as the wording of individual items is not context or disease specific, further research is needed to assess the transferability of adapted versions of the SCQ.

- The SCQ allows healthcare providers, practice settings, institutions and healthcare systems to routinely assess and improve patients' experiences of compassion, while providing researchers the means to conduct empirical research on this important care construct.

\section{BACKGROUND}

Compassion is 'a virtuous response that seeks to address the suffering and needs of a person through relational understanding and action'. Increasingly, compassion is considered a key indicator of quality care by patients, families, medical associations, ${ }^{23}$ policy makers, healthcare organisations and governments. ${ }^{4-9}$ In addition to addressing each of the goals of the Framework for Healthcare Improvement, ${ }^{10}$ recent research has reported that compassion is positively associated with a variety of patient-reported outcomes, the alleviation of healthcare provider (HCP) burnout, positive health 
outcomes and improved quality of care ratings. 4 6 11-13 Deficiencies in compassion are associated with increased patient complaints, malpractice suits, healthcare costs, non-disclosure of health information, adverse medical events and patient mortality. ${ }^{4-8} 12-15$ Notably, a systemic lack of compassion has been identified as a common and central factor in a number of high-profile national reviews of healthcare failures. ${ }^{4-71215}$

Although compassion in healthcare is increasingly mandated by governments and given credence by healthcare organisations, a persistent barrier to improving compassion cited in the literature, has been the lack of a valid and reliable patient-reported measure of compassion. ${ }^{5} 11$ 15-17 While measures have begun to emerge to address this gap, a recent systematic review revealed that existing measures have significant limitations, including but not limited to: insufficient evidence of internal consistency; validity; test accuracy; reliability and sensitivity. $^{11} 1819$ Currently, there are no patient-reported compassion measures that: (a) adhere to measure development guidelines ${ }^{20-22}$; (b) were developed from an empirical model of the construct of interest; (c) adequately assess the credibility and transferability of the measure across patient populations and importantly $(d)$ engaged patients across each stage of measure development. ${ }^{11}$ The objective of the current study was to develop and validate a psychometrically rigorous, patient-reported measure of compassion for use in clinical practice and research.

Before embarking on the current study we: conducted a scoping review of compassion ${ }^{4}$; defined and developed an empirical model of compassion from the perspective of patients ${ }^{1}$ (see online supplemental figure S1) demarcated compassion from sympathy and empathy, ${ }^{23}$ and validated the model among $\mathrm{HCPs}^{16}$ (see online supplemental figure S2). The current study began with qualitative interviews with patients to establish the transferability of the model across our study populations and focus groups with HCP, educators and administrators $(n=24)$ to determine the feasibility, challenges, facilitators and clinical utility of the proposed measure (see online supplemental table S1). The results of this first study stage, ${ }^{24}$ along with the findings of our afore-mentioned literature review and model development directly informed the item generation stage of the study, ${ }^{25}$ in accordance with development guidelines. ${ }^{20-22}$ Finally, the content validity of the draft measure was established through a Delphi process with international subject matter experts and patient advisors, along with cognitive interviews with patients. ${ }^{26}$ In addition to revising the initial item pool, this initial validation phase established the: relevancy of each item; representativeness of items to the construct and associated domain of the compassion model; clarity and readability of each item; and the relevancy of the recall period and the response scale. This article reports on the results of the exploratory and confirmatory factor analysis (CFA) stages of this large multi-centred study, producing the final version of the Sinclair Compassion Questionnaire (SCQ).
METHODS

\section{Patient and public involvement}

Patients and former patients, who comprised the studies patient advisory group, were involved in the design, or conduct, or reporting, or dissemination plans of this study-including assessing the transferability of the patient model, item development, cognitive interviews and measure development. ${ }^{123-26}$

\section{METHODS: PHASE 1-EXPLORATORY FACTOR ANALYSIS Phase 1: study design and study population}

To assess the structure and reliability of the draft 54-item compassion measure that emerged from the content validity stage ${ }^{26}$ using EFA, 303 participants were recruited between February 2018 and September 2018 from four care settings in two Canadian cities: acute care $(n=105)$, home care $(n=13)$, long term care (LTC) $(n=130)$ and hospice $(n=55)$ (see online supplemental table S2). Eligibility criteria included: being age 18 years or older; being able to read and speak English; living with a lifelimiting illness (eg, cancer, chronic obstructive pulmonary disease, coronary heart disease, dementia); having the cognitive capacity to complete the study (as assessed by the healthcare team) and being able to provide informed consent. Eligible patients/residents $(n=713)$ were identified by a member of the healthcare team and informed of the study. Patients/residents were not referred to the study if they were cognitively impaired, unable to provide informed consent, or too ill to participate. Of the 713 eligible participants informed about the study, 209 (29.3\%) chose not to or could not participate for various reasons (too tired $n=43$; not interested $n=92$; language barrier $n=8$; discharged/deceased $n=14$; other $\mathrm{n}=13$; undisclosed $\mathrm{n}=39$ ). Of the remaining 504 patients / care residents who agreed to speak to a Research Assistant (RA) about participating, 201 (39.9\%) chose not to or could not participate. The remaining 303 participants (response rate $=42.5 \%$ ) were provided with further study details by the RA, who was not a member of the healthcare team and assured patients that their responses would not be shared with members of their healthcare team. The protocol was administered within 24 hours after obtaining written informed consent (see online supplemental table S2). Participants were asked to selfadminister the questionnaire via tablets (adjustable font size) using REDCap survey software, or on paper if preferred. If a participant had difficulty completing the questionnaire (eg, poor eyesight or weakness), the RA administered the questionnaire.

\section{Phase 1: study procedures}

Study participants were administered the draft 54-item compassion measure asking them to rate their experience of compassion from their HCPs over the past 7 days using a 5-point Likert scale of agreement ( $1=$ strongly disagree, 2=disagree, $3=$ neutral, 4=agree, $5=$ =strongly agree). Additionally, participants were asked to rate the importance of 
each of the 54 items on a 5-point Likert scale ( $1=$ not at all important, $2=$ notvery important, $3=$ somewhat important, $4=$ very important, $5=$ extremely important). Test-retest reliability was assessed by having the same RA re-administer the 54-item measure to 65 consenting participants within a 24 -hour period. ${ }^{22}$

\section{Phase 1: data analysis}

All data analyses were completed using SPSS Statistical Software (V.24). In an effort to achieve maximum measurement stability with the fewest number of items, the set of 54 initial items emerging from initial validity testing, ${ }^{26}$ was further reduced based on the test-retest reliability results. Items below an intraclass correlation coefficient (ICC) threshold of 0.70 , the upper end of 'good' ICC values, ${ }^{27}$ were considered less stable across testing sessions and were not considered optimal items. They were therefore eliminated, a strategy that has been used in other scale development work. ${ }^{28} 29$ Exploratory factor analysis (EFA) using a principal axis factor (PAF) extraction with direct oblimin rotation was used to explore the underlying structure of the data. ${ }^{30}$ Pairwise deletion was used for any missing values. Parallel analysis was used to assess the number of retained factors. ${ }^{31}$ Refinement of the compassion measure occurred by examining factor loadings, domain coverage within the Patient Compassion Model (see online supplemental figure $\mathrm{S} 1$ ), ${ }^{1}$ and internal consistency.

\section{RESULTS: PHASE 1-EFA}

Participants took approximately $30 \mathrm{~min}$ to complete the draft 54-item SCQ and importance questions, regardless of whether it was self-administered $(\mathrm{n}=68)$ or RA facilitated $(n=234)$, with $71.9 \% \quad(n=217)$ of the questionnaires being administered by tablet (see online supplemental table S2). Initial test-retest assessment of the 54 items, resulted in five items achieving an ICC below 0.70 and were therefore discarded (see online supplemental table S3). In order to make an objective decision regarding item retention, the remaining 49 items were subjected to an EFA, using PAF to identify which of a number of alternate worded items to retain (eg, my HCP showed genuine concern vs genuine interest), while removing the lower loaded factor alternate. To first ensure that the data were suitable for factor analysis we conducted the Kaiser-MeyerOlkin (KMO) measure of sampling adequacy (0.976) and Bartlett's test of sphericity $\left(\chi^{2}=137797.78, \mathrm{df}=1176\right.$, $\mathrm{p}<0.001)$. The minimum threshold for acceptability for $\mathrm{KMO}$ values in conducting factor analysis is $<0.50,{ }^{32}$ with a significant Bartlett's test of sphericity. ${ }^{33}$ These values indicated that our data were optimal for EFA. The PAF and subsequent analysis of factor loadings between alternate worded items resulted in the removal of 11 redundant items (see online supplemental table S4).

To ensure that the remaining 38 items were adequate for factor analysis, the KMO measure of sampling adequacy (0.974) and Bartlett's test of sphericity $\left(\chi^{2}=10023.92\right.$, $\mathrm{df}=703, \mathrm{p}<0.001)$ were again assessed and deemed appropriate. The second PAF was conducted (see online supplemental table S5), which resulted in the extraction of a single factor that explained approximately $57 \%$ of the variance, yielding an overall Cronbach's alpha of 0.979 . The decision to extract a single factor was determined through the use of parallel analysis, rather than eigenvalues greater than one, or scree plots, due to the tendency of the latter to result in over-extraction. ${ }^{31}$

To determine the optimal number of measure items, balancing maximal reliability and clinical feasibility, the measure was further refined based on factor loadings, internal reliability and domain coverage within the Patient Compassion Model (see online supplemental figure S1). ${ }^{1}$ Adhering to methodological guidelines, ${ }^{30}$ the Cronbach's alpha for the first four items that loaded highest onto this single factor was calculated and additional items were loaded sequentially, one-by-one, to determine the reliability of the items collectively until all 38 items were loaded to assess the diminishing returns of additional items (figure 1). Through an iterative consensus process among the research team, a cut-off of 15 items with the highest factor loadings was determined as optimal, as it yielded a Cronbach's alpha of 0.96 , while also providing sufficient domain coverage (see online supplemental figure $\mathrm{S} 1$ ), such that at least two items for each domain were included. These methodological decisions were based on an array of considerations, including psychometric soundness, the pragmatics of scale length and existing theoretical considerations, and are consistent with healthcare measure development recommendations. ${ }^{30}$ Although not explicitly a part of the selection process for these cuts, our decision was further supported by patients' mean importance ratings for the 15 -items, which remained high, ranging from (SD) 4.08 (0.75) to 4.30 (0.60) (table 1). Descriptive statistics on the 15-item SCQ including means, SD, skewedness, kurtosis, range, test-retest reliabilities and theoretical domain coverage were also assessed (tables 1 and 2).

\section{METHODS: PHASE 2-CFA}

\section{Phase 2: study design and study population}

Between February 2019 and September 2019, a new sample of 330 participants were recruited to confirm the factor structure obtained at the EFA stage and test convergent and divergent validity, using valid and reliable measures (see online supplemental table S6). Participants were recruited from acute care $(n=109)$, hospice $(\mathrm{n}=82)$ and LTC settings $(\mathrm{n}=139)$, across two Canadian cities (see online supplemental table S2). Homecare patients were excluded in this phase. The 7-day recall period in the question stem proved to be problematic, as many homecare patients did not require or receive an in-person visitation from a member of the homecare team over the course of a week. Of the 710 eligible (same criteria as phase 1) individuals, $176(24.8 \%)$ chose not to, or could not, participate for various reasons (too 


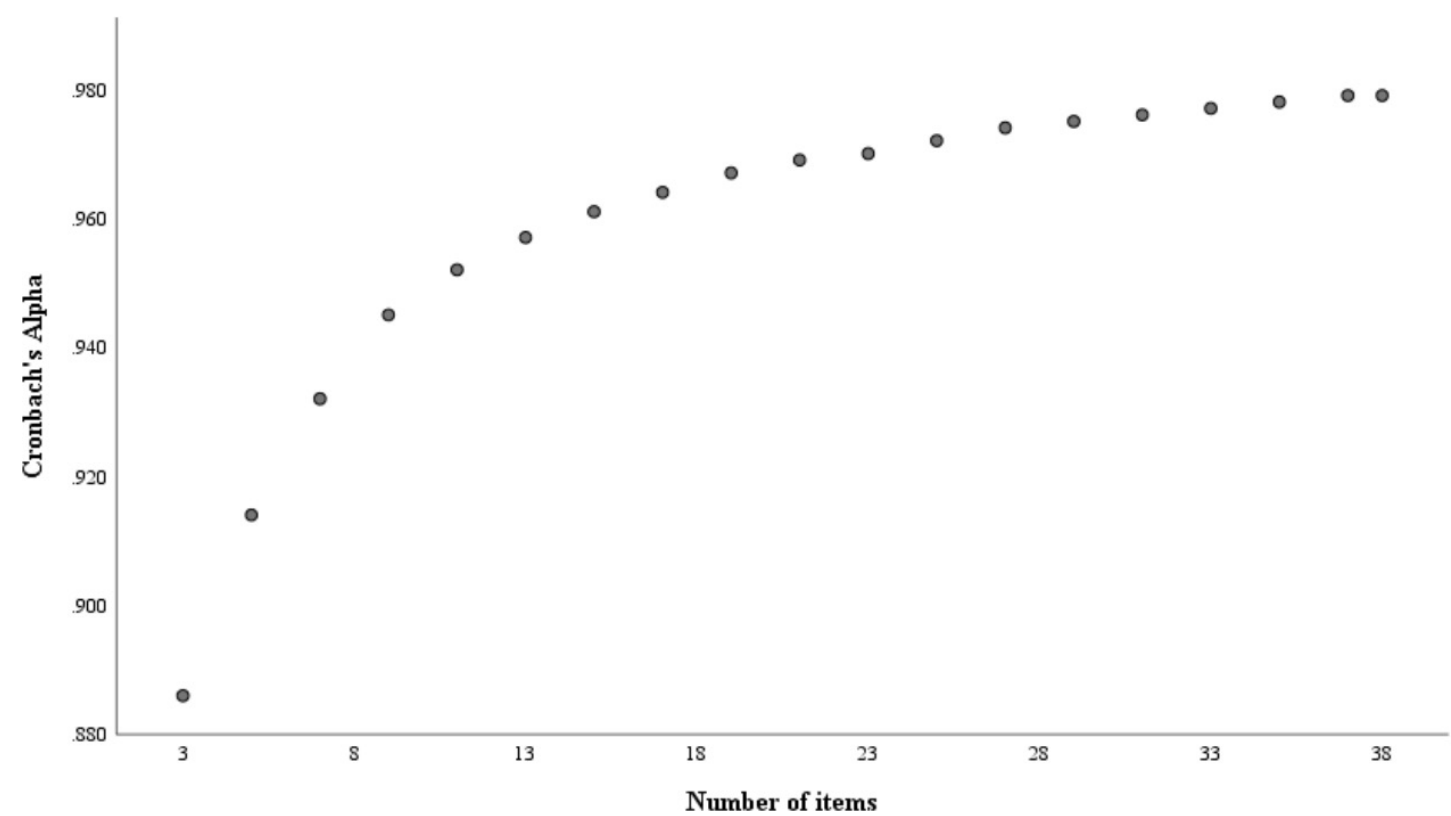

Figure 1 Compassion measure number of items against internal consistency reliability coefficients (Cronbach's alpha).

tired $n=37$; not interested $n=79$, language barrier $n=16$; discharged/deceased $\mathrm{n}=5$; other $\mathrm{n}=22$; undisclosed $\mathrm{n}=17$ ), after a member of their healthcare team gauged their initial interest in participating. Of the remaining 534 patients/care residents who agreed to speak to an RA about the study, $204(38.2 \%)$ chose not to, or could not, participate in the study, resulting in 330 participants (response rate $=46.5 \%)$ being administered the phase 2 protocol (see online supplemental table S2).

\section{Phase 2: study procedures}

Study participants were administered the 15-item SCQ, which was assessed at a Flesch-Kincaid grade level 8.
Compassion was also assessed using the 12-item Schwartz Center Compassionate Care Scale (SCCCS). ${ }^{34}$ The SCCCS question stem ('During your recent hospitalization, how successfully did your doctor ...') was modified to align with the current study's recall period and multi-provider focus ('In thinking about your Healthcare Providers over the past seven days, how successfully did they ...'). Next, symptom distress was assessed using the 9-item revised Edmonton Symptom Assessment Scale (ESAS-r). ${ }^{35} 36$ Finally, patient satisfaction was assessed using the 13-item PICKER Patient Experience Questionnaire (PPEQ). ${ }^{37}$ It was hypothesised that scores on the SCCCS would be

Table 1 Descriptive statistics for the final 15-item Sinclair Compassion Questionnaire based on phase 1 (exploratory factor analysis) sample

\begin{tabular}{lllllllll}
\hline Item & N & Min & Max & Mean & SD & Skewness & Kurtosis & $\begin{array}{l}\text { Mean importance } \\
\text { rating (SD) }\end{array}$ \\
\hline Feel cared for & 303 & 1 & 5 & 4.15 & 0.76 & -0.95 & 1.70 & $4.24(0.68)$ \\
Genuine concern & 302 & 1 & 5 & 4.12 & 0.75 & -0.82 & 1.10 & $4.20(0.68)$ \\
Communicated sensitive & 303 & 1 & 5 & 4.14 & 0.74 & -0.77 & 0.98 & $4.17(0.64)$ \\
\hline Attentive & 303 & 1 & 5 & 4.14 & 0.72 & -0.70 & 1.08 & $4.30(0.60)$ \\
Provided comfort & 303 & 1 & 5 & 4.07 & 0.73 & -0.89 & 1.68 & $4.23(0.62)$ \\
Very supportive & 301 & 1 & 5 & 4.18 & 0.66 & -0.63 & 1.46 & $4.19(0.62)$ \\
Provided care & 302 & 2 & 5 & 4.19 & 0.65 & -0.65 & 1.21 & $4.25(0.58)$ \\
Spoke with kindness & 303 & 1 & 5 & 4.24 & 0.69 & -1.02 & 2.79 & $4.27(0.60)$ \\
\hline Saw as person & 302 & 1 & 5 & 4.09 & 0.78 & -0.87 & 1.03 & $4.23(0.64)$ \\
Behaved in caring way & 300 & 2 & 5 & 4.20 & 0.66 & -0.59 & 0.77 & $4.28(0.57)$ \\
\hline Really understood needs & 303 & 1 & 5 & 3.91 & 0.88 & -0.76 & 0.48 & $4.24(0.66)$ \\
\hline Good relationship & 303 & 1 & 5 & 4.17 & 0.75 & -0.88 & 1.24 & $4.28(0.56)$ \\
\hline See my perspective & 303 & 1 & 5 & 3.85 & 0.89 & -0.55 & 0.05 & $4.08(0.75)$ \\
\hline Warm presence & 302 & 2 & 5 & 4.11 & 0.70 & -0.51 & 0.34 & $4.15(0.65)$ \\
\hline Sincere & 303 & 2 & 5 & 4.15 & 0.71 & -0.61 & 0.40 & $4.21(0.55)$ \\
\hline
\end{tabular}


Table 2 Test-retest reliability and theoretical domain coverage for the final 15-item Sinclair Compassion Questionnaire

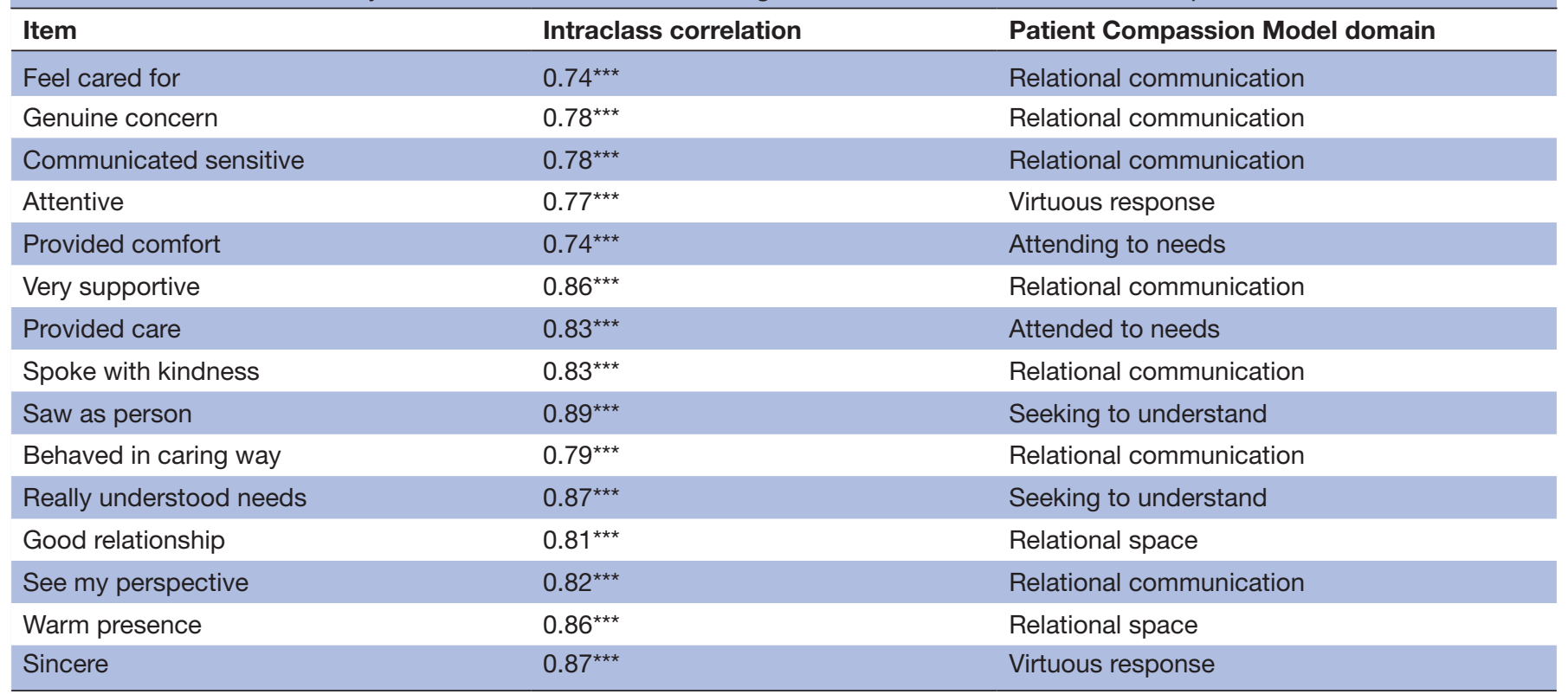

${ }^{* * *} \mathrm{p}<0.001,{ }^{* *} \mathrm{p}<0.01,{ }^{*} \mathrm{p}<0.05$

Mean time interval $=24$ hours.

strongly and positively associated with the SCQ scores, whereas scores from the discriminant constructs such as symptom distress (ESAS-r) and patient satisfaction (PPEQ) would be positively associated with the SCQ, but less so.

\section{Phase 2: data analysis}

The initial factor structure identified via EFA was tested through CFA, using AMOS (V.24) with missing data excluded listwise. Factor loadings were explored, and global model fit was evaluated by examining model $\chi^{2}$, comparative fit index (CFI), root-mean-square-error of approximation (RMSEA) and standardised root-meansquared residual (SRMR). Ideal fit criteria for these indices include non-significant $\chi^{2}$ tests, CFI above 0.95, SRMR values less than $0.08^{38} 39$ and RMSEA values below 0.08 . ${ }^{40}$ It is important to note, that model $\chi^{2}$ tests are highly sensitive to minor deviations from perfect model fit and sample size, and are often found to be statistically significant despite an excellent fitting model..$^{38}$ Potential areas of misfit were therefore improved by including several covariances between residuals in the measurement structure. Item response theory (IRT) was also used to evaluate the relationships between the latent construct of compassion and the items intended to measure the construct. Missing values for observation were excluded from the analysis and did not contribute to the likelihood estimates in the IRT analysis.

Validity evidence between overall scores from the SCQ and those from the measures of convergent and discriminant validity were explored using Pearson correlations. For exploratory purposes, potential differences in overall SCQ scores as a function of study sites (acute care, hospice and LTC) and sociodemographic variables were examined using an analysis of variance (ANOVA). Any missing values for these analyses were treated with pairwise deletion.

\section{RESULTS: PHASE 2-CFA AND RELIABILITY}

Participants took approximately 20 min to complete the protocol, with $259(78.5 \%)$ of the participants requiring the assistance of an RA. The 15-item SCQ took participants between 3 and 5 min to complete, with $224(67.9 \%)$ being completed via tablet and $21.6 \%$ being self-administered (see online supplemental table S2). Only participants with complete data on the SCQ compassion items were included in the CFA ( $\mathrm{N}=327)$.

Cronbach's alpha for the 15-item measure in phase 2 was 0.96 . Given the single factor solution revealed at the EFA stage, a single latent compassion factor was specified with loadings from each of the 15 items. Initial model estimation revealed strong standardised factor loadings ranging between 0.75 and 0.86 , with some global fit indices initially being unsatisfactory. Global fit improved by adding covariances to the model, yielding standardised factor loadings that remained strong, between 0.76 and 0.86 (figure 2). Overall, these results support a single factor of compassion.

IRT analyses indicated that the SCQ precisely measures compassion across the wide range of patients' experiences with their HCPs. The average marginal reliability of the SCQ was 0.85 . The parameter estimates were reasonable and their standard errors were small. For each of the SCQ items, the item discrimination parameter was statistically significant, implying that each item can differentiate between individuals with different ratings of compassion. Further analysis indicated that participants who felt that 


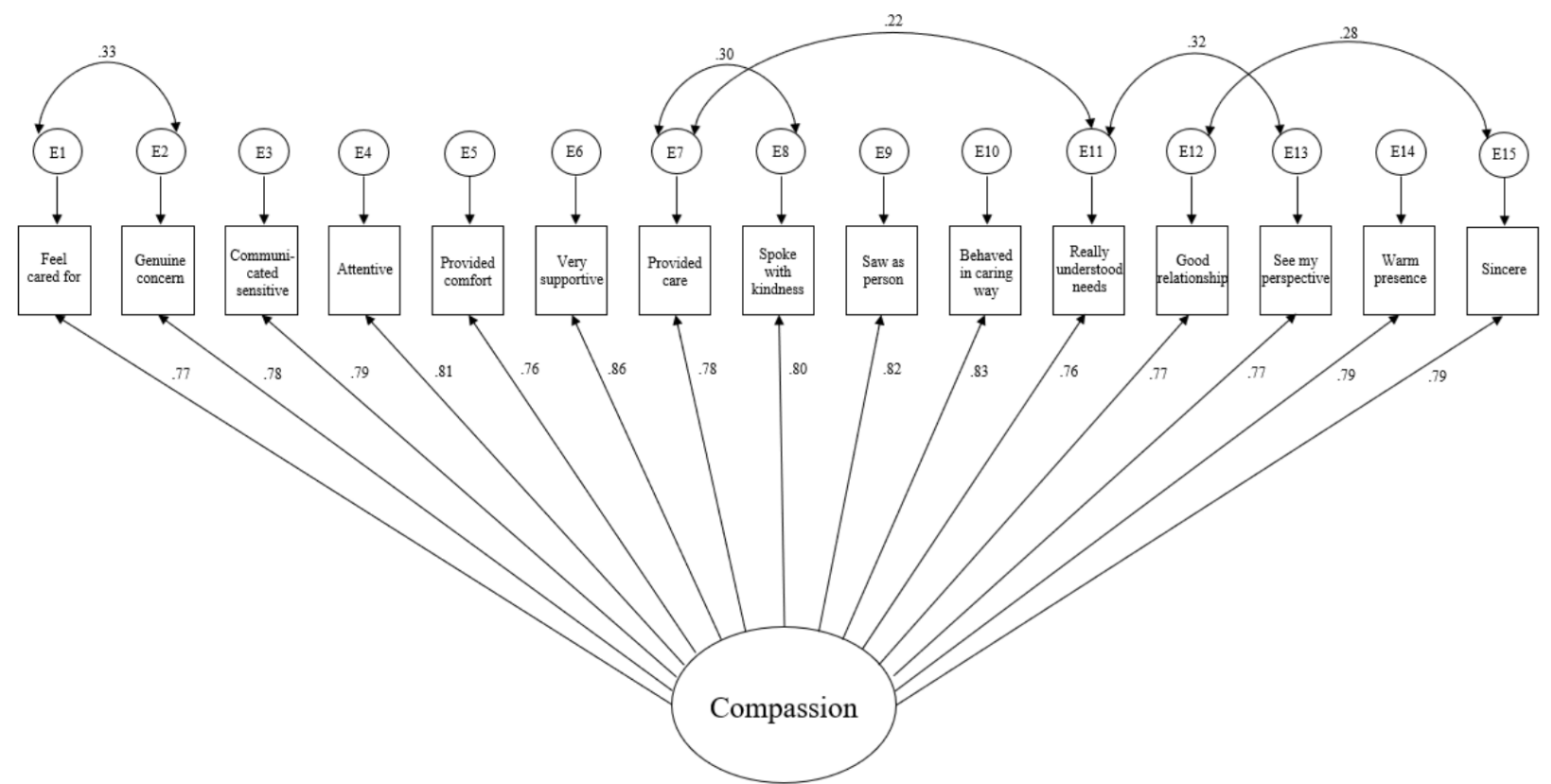

Figure 2 Final confirmatory factor analysis model for the Sinclair Compassion Questionnaire. Standardised loadings reported. Fit indices: $\chi^{2}=219.38, p<0.001$, comparative fit index $=0.97$, root-mean-square-error of approximation $=0.07$, standardised rootmean-squared residual $=0.03$. Cronbach's alpha $=0.96$.

their HCPs were very compassionate were highly likely to select 'strongly agree' to relevant SCQ items, while those who believed that their HCPs were less compassionate were likely to select the appropriate response; providing further confidence in the reliability and precision of the SCQ. Descriptive statistics for the final 15-item SCQ based on phase 2 (CFA) sample can be referred to in table 3 .

When assessing convergent validity, the SCCCS $(\alpha=0.97)$ and the SCQ were strongly positively correlated, $r=0.75$, $\mathrm{p}<0.001$, providing support that the SCQ taps into the construct of compassion. When assessing for divergent validity, the SCQ and the PPEQ $(\alpha=0.88)$ were positively correlated with moderately high strength $(r=0.60$, $\mathrm{p}<0.001$ ). This provides evidence that the SCQ is related to, but sufficiently distinct from, patient satisfaction. Finally, the relationships between the SCQ and each individual ESAS-r symptom were assessed for evidence of divergent validity. The SCQ was significantly associated with depression and well-being, with individuals who reported lower depression or greater well-being having higher compassion scores. Compassion was, however, weakly and

Table 3 Descriptive statistics for the final 15-item Sinclair Compassion Questionnaire based on phase 2 (confirmatory factor analysis) sample

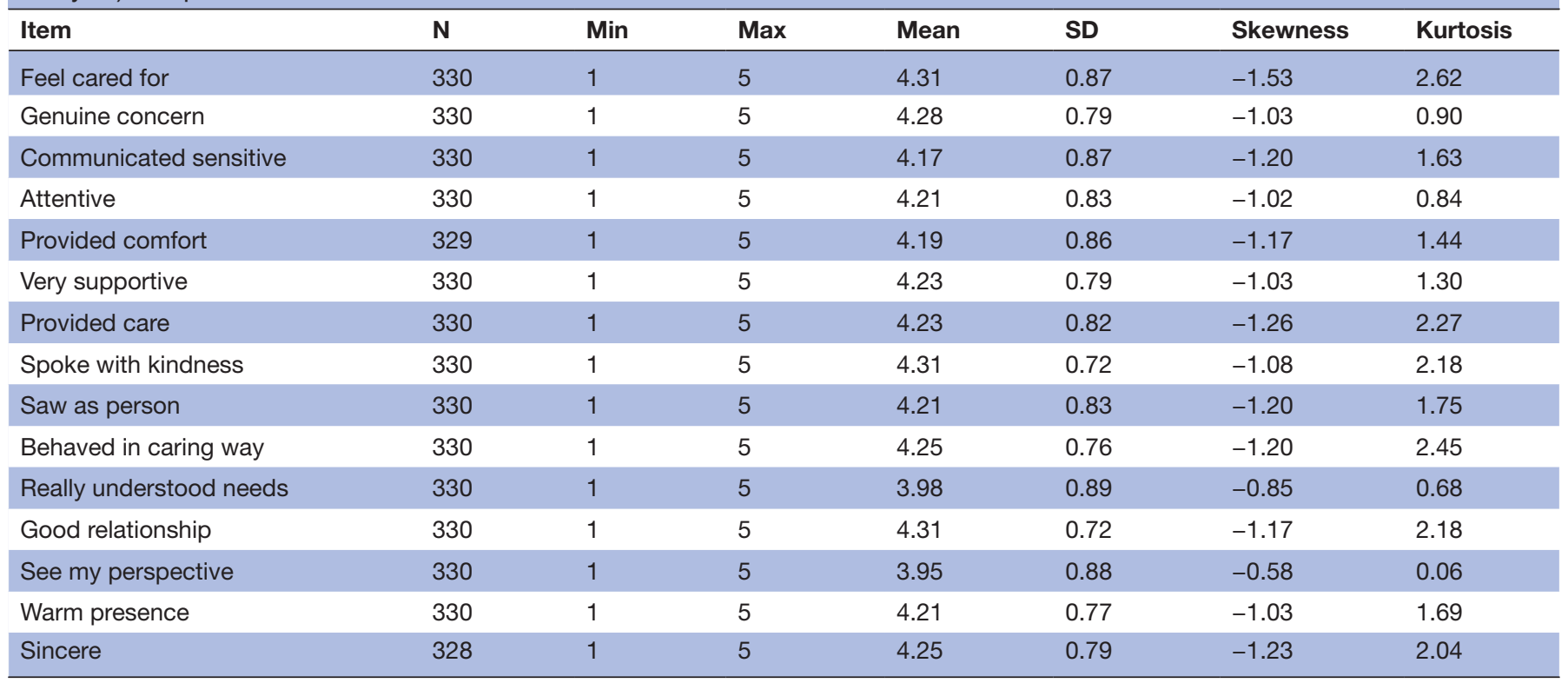


negatively associated with depression $(\mathrm{r}=-0.13, \mathrm{p}=0.02)$, and well-being $(\mathrm{r}=-0.17, \mathrm{p}=0.002)$, with higher depression scores on the ESAS-r indicating greater depression and higher well-being scores indicating worse well-being. No additional relationships between the SCQ and other symptoms measured by the ESAS-r (eg, pain, fatigue, anxiety, sleep, etc) reached statistical significance. This provides support that the SCQ construct of compassion is distinct from symptom distress.

To further validate the SCQ, we assessed whether SCQ scores varied as a function of demographic variables or care setting. ANOVAs yielded no significant mean differences on the SCQ by gender $(\mathrm{p}=0.784)$, marital status $(\mathrm{p}=0.403)$, education $(\mathrm{p}=0.240)$, ethnicity $(\mathrm{p}=0.551)$ or spirituality/religiousness $(\mathrm{p}=0.589)$. A weak, negative correlation between age and reported SCQ scores was identified $(\mathrm{r}=-0.13, \mathrm{p}=0.021)$. Significant differences emerged between compassion scores and care location, $\mathrm{F}(2,327)=16.62, \mathrm{p}<0.001$. Post-hoc Tukey's tests revealed that individuals in acute care $(\mathrm{M}=4.39)$ reported significantly higher $(\mathrm{p}<0.001)$ levels of compassion than those in LTC $(\mathrm{M}=3.97)$. Similarly, participants in hospice $(\mathrm{M}=4.37)$ reported significantly greater levels of compassion $(\mathrm{p}<0.001)$ than those in LTC. No differences were found between those in acute care and hospice settings $(p=0.975)$. Given that compassion scores were influenced by age and care location, exploratory follow-up analyses were conducted to examine (a) potential age differences by care location and (b) differences in compassion by care location when statistically controlling for age. Age differed by care location, $\mathrm{F}(2,317)=27.55$, $\mathrm{p}<0.001$, with acute care participants $(\mathrm{M}=66.89)$ being significantly younger than LTC residents $(\mathrm{M}=79.37, \mathrm{p}<0.001)$ and hospice patients $(\mathrm{M}=75.61, \mathrm{p}<0.001)$. However, when examining compassion by care location statistically controlling for age, results remained consistent with those reported earlier, $F(3,316)=11.65, \mathrm{p}<0.001$, with compassion being higher in acute care and hospice settings versus LTC, ps $<0.001$. A separate secondary CFA analysis was also conducted to assess the validity and reliability of a 5-item short-form version (SCQ-SF), in order to provide further flexibility and utility to clinicians and survey administrators wanting to embed a measure of compassion in their clinical assessments and patient/family surveys, without compromising psychometric rigour (see online supplemental table S7 and figure S3).

\section{INSTRUCTION MANUAL, SCORING GUIDELINES AND VERSIONS OF THE SCQ}

Scoring of the SCQ is performed by calculating the mean score of all the items, with an overall score being indicative of greater reported compassion. The SCQ, a detailed instruction manual including administration, data entry, response coding and scoring instructions is available from the authors or at www.compassionmeasure.com. The SCQ French version (Questionnaire de Compassion Sinclair) is also available, with a Spanish version forthcoming.

\section{DISCUSSION}

This study validated a patient-reported measure of compassion, the SCQ, providing researchers, clinicians, survey administrators and healthcare organisations a clinically informed, patient-reported measure of compassion that has strong initial evidence supporting its validity and reliability. The SCQ contains items that cover patients' experiences of compassion within each of the theoretical domains of the Patient Compassion $\mathrm{Model}^{1}$ with our results showing that these domains are subsumed under a single latent construct of compassion. These results are a defining feature of reflective measures, ${ }^{25}$ whereby individual items each reflect the underlying construct, underscoring the necessity of conducting foundational

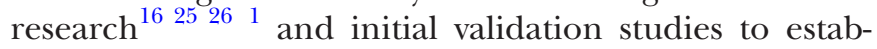
lish construct validity ${ }^{24-26}$ —an essential, but overlooked stage in the development of compassion measures ${ }^{11}$ and measure in general. ${ }^{19-21}$ As a result, the SCQ has excellent internal consistency (Cronbach's alpha of 0.96) and testretest reliability (ranging from 0.74 to 0.89 ).

Results demonstrate that the SCQ overcomes the inherent limitations of previous compassion measures in healthcare. ${ }^{11181934}$ Notably, the SCQ is a patient-reported measure of compassion (vs a clinician or proxy assessment) that rigorously adheres to measure development guidelines and uses appropriate response scales for the construct of interest (agreement vs frequency or satisfaction). ${ }^{19} 212526$ Further, the perspectives of patients were incorporated across each stage of this study, ${ }^{124-26}$ fortifying our foundational patient-centred research that defined the construct of interest and its associated domains, including how compassion is delineated and preferred over similar constructs such as sympathy and empathy-which are often conflated within existing compassion measures. ${ }^{23}$ This is critical, as while compassion subsumes and enhances elements of empathy, patient-centred care and clinical communication, it is a separate construct that includes action and the virtues of HCPs-providing a multimodal and optimal effect on various patient outcomes that do not come at the cost of HCP well-being. ${ }^{13} 41$

In addition, the SCQ provides healthcare organisations with the means to assess patient experiences of compassion alongside other quality care indicators, patient experience measures and satisfaction surveys such as the PPEQ. ${ }^{37}$ Reporting patients' compassion scores at the unit, institutional and systems-level allows researchers and health administrators to identify benchmarks and analyse associations between compassion and other key variables (eg, patient satisfaction, workplace well-being, adverse medical events and between patient groups). Further, a valid patient-reported compassion measure provides investigators the opportunity to study distributions of compassion preferences across various populations and care settings. While the 15-item SCQ is brief and easy to complete, the 5-item SCQ-SF provides additional flexibility for clinicians who want to assess and improve compassion in their professional practice. 
Secondary analysis revealed compassion scores were significantly lower among those in LTC and were weakly and negatively correlated with age. After controlling for age, a negative statistically significant difference remained between SCQ scores in LTC and other settings. Possible explanations for lower SCQ scores in LTC may be due to differing staff-patient ratios, levels of acuity or suffering, or differences in practice culture; however, further research is required to examine this difference.

\section{Strengths and limitations}

This study is not without limitations. First, while the establishment of the recall period was determined by the construct being measured; measure development guidelines, and was validated through a Delphi process with international SMEs and cognitive interviews with patients, ${ }^{20} 2526$ the SCQ may have lost some specificity, as we chose a 7-day recall period that asked respondents to consider all interactions with their HCPs during that period. This decision was intentional and evidenceinformed, as patients, members of our HCP focus groups, subject matter experts and members of our patient advisory group felt that a 7-day period was most appropriate for our study populations. ${ }^{1626}$ The same data informed our decision to measure patients overall experience of compassion from all HCPs they interacted with, rather than care from a single HCP at a specific timepoint-a decision that is further supported by guidelines for measuring quality care within the patient experience. ${ }^{191623}$ Second, while we anticipate that the SCQ is transferable to other patient populations and while we validated the measure across diverse care settings, ${ }^{24}$ in sampling patients with a life limiting illness, the generalisability of the measure to other patient populations requires further research, for example, in the general population, younger patients and other healthcare contexts. Third, while we attempted to validate the measure within a homecare setting, the question stem proved to be problematic, resulting in this cohort being removed from the CFA stage of the study. While it is anticipated that the SCQ is adaptable to other settings, as the wording of individual items is not context or disease specific, further research is needed to assess the validity of adapted versions of the SCQ with modified question stems. Fourth, although the strength of the SCQ as a brief, patient-reported measure addresses many of the limitations of previous measures, due to the acuity of our patient population, most patients required the aid of an RA to complete the protocol (78\% in both study phases). As such, when patients are unable to self-administer the SCQ, we recommend that it be administered by someone other than the patients' HCPs (eg, a patient care manager or RA) in order to mitigate response and social desirability bias. Fifth, in slightly modifying the recall period and the question stem of the measures we used for convergent and divergent validity with the SCQ, this may have influenced the fidelity and results from these measures (see online supplemental table S6).
While the initial evidence for construct validity of the SCQ is encouraging, additional evidence is needed to be establish its responsiveness, interpretability and criterion validity.

\section{CONCLUSIONS}

The SCQ is a reliable and valid patient-reported compassion measure for research and practice. The SCQ will allow HCPs, practice settings, institutions and healthcare systems to routinely assess and improve compassion, while providing researchers the means to conduct empirical research on this important care construct.

\section{Author affiliations}

${ }^{1}$ Faculty of Nursing, University of Calgary, Calgary, Alberta, Canada

${ }^{2}$ Compassion Research Lab, University of Calgary, Calgary, Alberta, Canada

${ }^{3}$ Department of Oncology, Cumming School of Medicine, University of Calgary, Calgary, Alberta, Canada

${ }^{4}$ College of Nursing, Rady Faculty of Health Sciences, University of Manitoba, Winnipeg, Manitoba, Canada

${ }^{5}$ Psychosocial Oncology and Cancer Nursing Research, IH Asper Clinical Research Institute, Winnipeg, Manitoba, Canada

${ }^{6}$ Department of Psychology, University of Calgary, Calgary, Alberta, Canada

Twitter Shane Sinclair @ShaneASinclair, Aynharan Sinnarajah @DrASinnarajah, Genevieve Thompson @DRThompson_GN and The COMPASS Research Team @ CRLinaction

Acknowledgements The authors wish to thank each of the patients who participated in this study and the members of our patient advisory group for their time, expertise and enthusiasm across each of the stages of developing the measure. We would also like to thank the research personnel who made this study a success: Reilly Campbell, Shaista Turner, Carolyn Campbell, Marilyn Lindquist, Katherine Cullihall, Ceceile Porter, Nicole Shead, and Courtney Teetaert (Research Assistants who supported data collection), Grace Perez (who provided statistical support), Dr. Devesh Oberoi (IRT Analysis), Melanie Paulin (French translator), and our clinical partners in both Winnipeg (St. Boniface Hospital, Riverview Health Centre, Deer Lodge Centre, Actionmarguerite, Saul and Claribel Simkin Centre, Bethania Mennonite Personal Care Home, Grace Hospice, Jocelyn House, WRHA Palliative Home Care) and Calgary (Intercare Chinook Hospice and Residential Care, Southwood Hospice and Residential Care, Rosedale Hospice, Rockyview \& Foothills Hospital Palliative Care Consult Service and Intensive Palliative Care Unit, Covenant Care Dulcina Hospice, Holy Cross Manor and St. Marguerite Residential Care), for securing site access and supporting our participant recruitment efforts. The Sinclair Compassion Questionnaire (SCQ), SCQ-Short Form (SCQ-SF) and other adaptions are available at www.compassionmeasure.com; by emailing the contact author directly or by emailing ipm@innovatecalgary.com.

Collaborators The COMPASS Research Team: Harvey Chochinov, Shelly Cory, Neil Hagen, Max Jajszczok, Leah Lechelt, Carlo Leget, Christina Puchalski, Patrick Quail, Lucy Selman.

Contributors SS conceptualised the study, obtained funding, developed, refined and determined the final wording of the SCQ items, oversaw data collection and analysis across sites in all stages of measure development, wrote the final manuscript and was responsible for all stages of the study. TFH conceptualised the study, obtained funding, developed, refined and determined the final wording of the SCQ items, oversaw data collection at the Winnipeg sites, contributed to data analysis and the final manuscript. CCM oversaw data analysis and interpretation in the EFA and CFA stages and contributed to the final manuscript. PJ coordinated the entire study, managed data across all sites, contributed to the development and refinement of the final wording of the SCQ items, contributed to data analysis and the final manuscript. $\mathrm{HB}$ conducted the data analysis and interpretation in the EFA and CFA stages and contributed to the final manuscript. SM contributed to the conceptualisation of the study, supported data collection at the Winnipeg sites and contributed to the final manuscript. GT contributed to the conceptualisation of the study, supported data collection at the Winnipeg sites and contributed to the final manuscript. AS contributed to the conceptualisation of the study, supported data 
collection at the Calgary sites, and contributed to the final manuscript. All authors reviewed and approved the final draft of the manuscript.

Funding This work was supported by the Canadian Institutes of Health Research, Project Scheme Grant (\#148543).

Competing interests None declared.

Patient consent for publication Not required.

Ethics approval This study was approved by the University of Calgary Conjoint Health Research Ethics Board (REB \#17-1854) and by the human research ethics committees at the Rady Faculty of Medicine, University of Manitoba (REB \#HS21470), St. Boniface Hospital and the Winnipeg Regional Health Authority.

Provenance and peer review Not commissioned; externally peer reviewed.

Data availability statement Data are available upon reasonable request. The datasets used and/or analysed during the current study are available from the corresponding author on reasonable request.

Supplemental material This content has been supplied by the author(s). It has not been vetted by BMJ Publishing Group Limited (BMJ) and may not have been peer-reviewed. Any opinions or recommendations discussed are solely those of the author(s) and are not endorsed by BMJ. BMJ disclaims all liability and responsibility arising from any reliance placed on the content. Where the content includes any translated material, BMJ does not warrant the accuracy and reliability of the translations (including but not limited to local regulations, clinical guidelines, terminology, drug names and drug dosages), and is not responsible for any error and/or omissions arising from translation and adaptation or otherwise.

Open access This is an open access article distributed in accordance with the Creative Commons Attribution Non Commercial (CC BY-NC 4.0) license, which permits others to distribute, remix, adapt, build upon this work non-commercially, and license their derivative works on different terms, provided the original work is properly cited, appropriate credit is given, any changes made indicated, and the use is non-commercial. See: http://creativecommons.org/licenses/by-nc/4.0/.

\section{ORCID iDs}

Shane Sinclair http://orcid.org/0000-0003-4542-9911

Thomas F Hack http://orcid.org/0000-0002-6913-8732

Cara C Maclnnis http://orcid.org/0000-0001-5418-1626

Harrison Boss http://orcid.org/0000-0003-4472-1048

Aynharan Sinnarajah http://orcid.org/0000-0002-7967-159X

Genevieve Thompson http://orcid.org/0000-0003-2558-9926

\section{REFERENCES}

1 Sinclair S, McClement S, Raffin-Bouchal S, et al. Compassion in health care: an empirical model. J Pain Symptom Manage 2016;51:193-203.

2 Canadian Medical Association. Code of ethics, 2004. Available: https://policybase.cma.ca/en/permalink/policy13937

3 American Medical Association. Code of medical ethics: principle 1, 2001. Available: https://www.ama-assn.org/sites/ama-assn.org/files/ corp/media-browser/principles-of-medical-ethics.pdf

4 Sinclair S, Norris JM, McConnell SJ, et al. Compassion: a scoping review of the healthcare literature. BMC Palliat Care 2016;15:1-16.

5 Francis R. Report of the mid Staffordshire NHS Foundation trust public inquiry. London: The Stationary Office, 2013.

6 Lown BA, Rosen J, Marttila J. An agenda for improving compassionate care: a survey shows about half of patients say such care is missing. Health Aff 2011;30:1772-8.

7 Willis L. Raising the bar: the shape of caring review. London: Health Education England, 2015.

8 The Beryl Institute. Consumer study on patient experience, 2018. Available: https://www.theberylinstitute.org/page/ PXCONSUMERSTUDY

9 Hanefeld J, Powell-Jackson T, Balabanova D. Understanding and measuring quality of care: dealing with complexity. Bull World Health Organ 2017;95:368-74.

10 Bodenheimer T, Sinsky C. From triple to quadruple AIM: care of the patient requires care of the provider. Ann Fam Med 2014;12:573-6.

11 Sinclair S, Russell LB, Hack TF, et al. Measuring compassion in healthcare: a comprehensive and critical review. Patient 2017;10:389-405.

12 Lown BA, Dunne H, Muncer SJ, et al. How important is compassionate healthcare to you? A comparison of the perceptions of people in the United States and ireland. J Res Nurs 2017;22:60-9.
13 Trzeciak S, Mazzarelli A. Compassionomics: the revolutionary scientific evidence that caring makes a difference. Pennsacola: Studer Group, 2019.

14 Reader TW, Gillespie A, Roberts J. Patient complaints in healthcare systems: a systematic review and coding taxonomy. BMJ Qual Saf 2014;23:678-89.

15 Maclean R. The Vale of leven Hospital inquiry. Edinburgh: APS Group, 2014.

16 Sinclair S, Hack TF, Raffin-Bouchal S. What are healthcare providers' understandings and experiences of compassion? the healthcare compassion model: a grounded theory study of healthcare providers in Canada. BMJ Open 2018;8:e019701.

17 Paterson R. Can we mandate compassion? Hastings Cent Rep 2011;41:20-3.

18 Roberts BW, Roberts MB, Yao J, et al. Development and validation of a tool to measure patient assessment of clinical compassion. JAMA Netw Open 2019;2:e193976.

19 Valderas JM, Ferrer M, Mendívil J, et al. Development of EMPRO: a tool for the standardized assessment of patient-reported outcome measures. Value Health 2008;11:700-8.

20 Reeve BB, Wyrwich KW, Wu AW, et al. ISOQOL recommends minimum standards for patient-reported outcome measures used in patient-centered outcomes and comparative effectiveness research. Qual Life Res 2013;22:1889-905.

21 Hinkin TR. A brief tutorial on the development of measures for use in survey questionnaires. Organ Res Methods 1998;1:1998:104-21.

22 Streiner D, Norman G, Cairney J. Health measurement scales. In: $A$ practical guide to their development and use. 5 edn. Oxford: Oxford University Press, 2015.

23 Sinclair S, Beamer K, Hack TF, et al. Sympathy, empathy, and compassion: a grounded theory study of palliative care patients' understandings, experiences, and preferences. Palliat Med 2017:31:437-47.

24 Sinclair S, Jaggi P, Hack TF, et al. Assessing the credibility and transferability of the patient compassion model in non-cancer palliative populations. BMC Palliat Care 2018;17:108.

25 Sinclair S, Jaggi P, Hack TF, et al. A practical guide for item generation in measure development: insights from the development of a patient-reported experience measure of compassion. J Nurs Meas 2020;28:138-56.

26 Sinclair S, Jaggi P, Hack TF, et al. Initial validation of a patientreported measure of compassion: determining the content validity and clinical sensibility among patients living with a life-limiting and incurable illness. Patient 2020;13:327-37.

27 Koo TK, Li MY. A guideline of selecting and reporting intraclass correlation coefficients for reliability research. J Chiropr Med 2016:15:155-63.

28 Hawker GA, Davis AM, French MR, et al. Development and preliminary psychometric testing of a new OA pain measure--an OARSI/OMERACT initiative. Osteoarthritis Cartilage 2008;16:409-14.

29 King M, Dinos S, Shaw J, et al. The stigma scale: development of a standardised measure of the stigma of mental illness. Br J Psychiatry 2007; 190:248-54.

30 Pett MA, Lackey NR, Sullivan JJ. Making sense of factor analysis: the use of factor analysis for instrument development in health care research. thousand oaks. CA: Sage, 2003.

31 Patil VH, Singh SN, Mishra S, et al. Efficient theory development and factor retention criteria: Abandon the 'eigenvalue greater than one' criterion. J Bus Res 2008;61:162-70.

32 Rice J, Jiffy L. Little Jiffy, mark IV. Educ Psychol Meas 1974;34:111-7.

33 Bartlett MS. Tests of significance in factor analysis. Br J Psychol 1950;3:77-85.

34 Lown BA, Muncer SJ, Chadwick R. Can compassionate healthcare be measured? the Schwartz center compassionate care Scale ${ }^{\mathrm{TM}}$. Patient Educ Couns 2015;98:1005-10.

35 Bruera E, Kuehn N, Miller MJ, et al. The Edmonton symptom assessment system (ESAs): a simple method for the assessment of palliative care patients. J Palliat Care 1991;7:6-9.

36 Watanabe SM, Nekolaichuk CL, Beaumont C. The Edmonton symptom assessment system, a proposed tool for distress screening in cancer patients: development and refinement. Psychooncology 2012;21:977-85.

37 Jenkinson C, Coulter A, Bruster S. The Picker patient experience questionnaire: development and validation using data from in-patient surveys in five countries. Int J Qual Health Care 2002;14:353-8.

38 Kline RB. Methodology in the social sciences. In: Principles and practice of structural equation modeling. 3 edn. New York: Guilford Press, 2011.

$39 \mathrm{Hu}$ Li-tze, Bentler PM. Cutoff criteria for fit indexes in covariance structure analysis: conventional criteria versus new alternatives. 
Structural Equation Modeling: A Multidisciplinary Journal 1999;6:1-55.

40 MacCallum RC, Browne MW, Sugawara HM. Power analysis and determination of sample size for covariance structure modeling. Psychol Methods 1996;1:130-49.
41 Sinclair S, Raffin-Bouchal S, Venturato L, et al. Compassion fatigue: a meta-narrative review of the healthcare literature. Int J Nurs Stud 2017b;69:9-24. 